\title{
A dyad of lymphoblastic lysosomal cysteine proteases degrades the antileukemic drug L-asparaginase
}

\author{
Naina Patel,, ${ }^{1}$ Shekhar Krishnan,, ${ }^{1}$ Marc N. Offman, ${ }^{2}$ Marcin Krol, ${ }^{2}$ Catherine X. Moss, ${ }^{3}$ \\ Carly Leighton, ${ }^{1}$ Frederik W. van Delft, ${ }^{1}$ Mark Holland, ${ }^{1}$ JiZhong Liu, ${ }^{1}$ Seema Alexander, ${ }^{1}$ \\ Clare Dempsey, ${ }^{1}$ Hany Ariffin, ${ }^{4}$ Monika Essink, ${ }^{5}$ Tim O.B. Eden, ${ }^{6}$ Colin Watts, ${ }^{3}$
}

Paul A. Bates, ${ }^{2}$ and Vaskar Saha ${ }^{1,6}$

\begin{abstract}
${ }^{1}$ Cancer Research UK Children's Cancer Group, Paterson Institute for Cancer Research, University of Manchester, Manchester, United Kingdom. 2Biomolecular Modelling Laboratory, Cancer Research UK London Research Institute, Lincoln's Inn Fields Laboratories, London, United Kingdom. ${ }^{3}$ Division of Cell Biology and Immunology, School of Life Sciences Research Biocentre, University of Dundee, Dundee, United Kingdom. ${ }^{4}$ Department of Paediatrics, University of Malaya, Kuala Lumpur, Malaysia. ${ }^{5}$ Medac GmbH, Wedel, Germany. ${ }^{6}$ Paediatric and Adolescent Oncology Unit, Christie Hospital, Manchester, United Kingdom.
\end{abstract}

\begin{abstract}
L-Asparaginase is a key therapeutic agent for treatment of childhood acute lymphoblastic leukemia (ALL). There is wide individual variation in pharmacokinetics, and little is known about its metabolism. The mechanisms of therapeutic failure with L-asparaginase remain speculative. Here, we now report that 2 lysosomal cysteine proteases present in lymphoblasts are able to degrade L-asparaginase. Cathepsin B (CTSB), which is produced constitutively by normal and leukemic cells, degraded asparaginase produced by Escherichia coli (ASNase) and Erwinia chrysanthemi. Asparaginyl endopeptidase (AEP), which is overexpressed predominantly in high-risk subsets of ALL, specifically degraded ASNase. AEP thereby destroys ASNase activity and may also potentiate antigen processing, leading to allergic reactions. Using AEP-mediated cleavage sequences, we modeled the effects of the protease on ASNase and created a number of recombinant ASNase products. The N24 residue on the flexible active loop was identified as the primary AEP cleavage site. Sole modification at this site rendered ASNase resistant to AEP cleavage and suggested a key role for the flexible active loop in determining ASNase activity. We therefore propose what we believe to be a novel mechanism of drug resistance to ASNase. Our results may help to identify alternative therapeutic strategies with the potential of further improving outcome in childhood ALL.
\end{abstract}

\section{Introduction}

On modern chemotherapeutic protocols, most children with acute lymphoblastic leukemia (ALL) show a rapid early response during the first 2 weeks of treatment. Though combinations of 10 or more drugs are used for over 2 years, those who show a slower response during this induction phase have a significantly worse outcome. The mechanisms of early response to therapy are not well understood, as even patients in the high-risk cytogenetic subtypes can show a rapid early response (1). In the United Kingdom and in many regimens used worldwide, 3 drugs are used during this phase, namely vincristine, steroids, and $\mathrm{L}$-asparaginase. There is evidence that $\mathrm{L}$-asparaginase potentiates the antileukemic effect of steroids (2) and when used intensively improves outcome (3-5). Thus, arguably, optimal usage of L-asparaginase is key to the early therapeutic response (6) and overall survival in childhood ALL.

Authorship note: Naina Patel and Shekhar Krishnan contributed equally to this work.

Conflict of interest: Monika Essink is an employee of Medac GmbH. All other authors have declared that no conflict of interest exists.

Nonstandard abbreviations used: AEP, asparaginyl endopeptidase; AEP-ABP, AEP activity-based probe; AEPi, AEP-specific inhibitor MV026630; ALL, acute lymphoblastic leukemia; AML, acute myeloid leukemia; ASNase, Escherichia coli asparaginase; CTSB, cathepsin B; CTSBi, CTSB-specific inhibitor CA074Me; Erwinase, Erwinia chrysanthemi asparaginase; LAMP1, lysosome-associated membrane protein 1; MD, molecular dynamics; PIC, protease inhibitor cocktail.

Citation for this article: J. Clin. Invest. 119:1964-1973 (2009). doi:10.1172/JCI37977.
L-asparaginase depletes asparagine (7). The dogma is that malignant lymphoblasts lack asparagine synthetase. In the absence of asparagine, they undergo apoptosis (8). A plasma L-asparaginase level of more than $100 \mathrm{U} / \mathrm{l}$ appears to correlate with adequate systemic asparagine depletion (9). Patients, however, show a wide variation in plasma levels after exposure to the drug (7). Though the majority achieve adequate levels on current therapeutic schedules, some patients, after an initial good response, fail to develop therapeutic levels on subsequent exposure. This is attributed to the development of silent neutralizing antibodies to the drug (10). Others have a rapid clearance of the drug on initial exposure but develop adequate levels subsequently $(7,11)$, and a few show insufficient asparaginase activity at any stage of treatment (Supplemental Table 1; supplemental material available online with this article; doi:10.1172/JCI37977DS1). Why this happens is not well understood. Florid hypersensitivity reactions can occur, necessitating withdrawal of the drug, though not all patients with hypersensitivity develop neutralizing antibodies and not all patients who develop neutralizing antibodies exhibit hypersensitivity $(12,13)$. Again, the reason why some patients develop allergy to L-asparaginase remains unknown. Other than the development of antibodies, resistance to L-asparaginase has been reported to occur by the production of asparagine synthetase by lymphoblasts (14-16) as well as marrow mesenchymal cells (17). Thus, optimization of therapy with L-asparaginase for all patients remains a challenge (18).

Allergic reactions pertain to the bacterial origin of the drug. There are 2 commercially available sources of L-asparaginase, one 

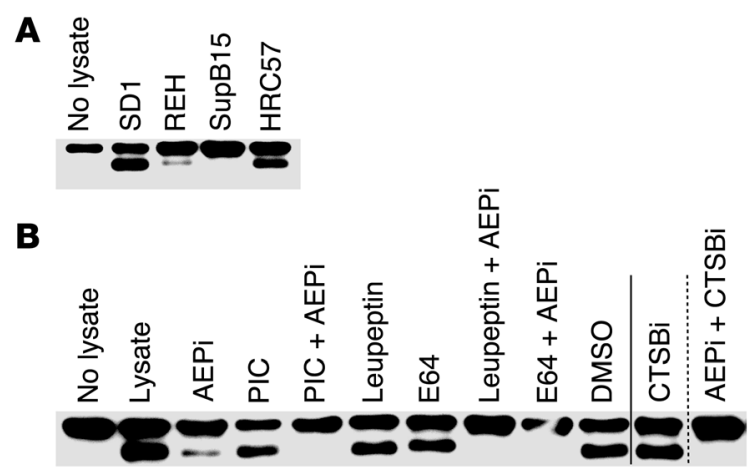

'inge

produced from Escherichia coli (ASNase) and the other from Erwinia chrysanthemi (Erwinase). The 2 products differ in half-life, activity, and antigenicity (19). The mechanisms of elimination of the drugs and the basis for the differences in activity are unknown. A polyethylene glycol-conjugated product of ASNase (ONCASPAR) has a significantly longer half-life, and in the UK, all children in treatment for childhood ALL receive ONCASPAR. The allergens/antigens for ASNase and Erwinase do not cross-react, and patients with clinical hypersensitivity to ASNase can safely receive Erwinase.

We recently reported that lymphoblasts of some ALL patients, primarily those in the high-risk cytogenetic subtypes, such as Philadelphia positive $\left(\mathrm{Ph}^{+}\right)$and iAMP21, express high levels of the lysosomal cysteine protease asparaginyl endopeptidase (AEP) (20). AEP has unusual specificity for cleavage after asparagine and aspartate, showing selectivity even among these residues (21). In the case of the tetanus toxin $\mathrm{C}$ fragment, AEP cleavage products present antigenic epitopes for MHC class II processing (22). $\mathrm{Ph}+$ patients are more likely to show a poor therapeutic response to ASNase (7), fall into the slower-response category (1), and develop hypersensitivity (V. Saha, unpublished observations). This led us to speculate that proteases present in lymphoblasts could cleave and inactivate bacterial asparaginases and potentiate the development of allergic reactions. Such a process would provide a cogent and unifying explanation of the variations in ASNase activity in patients and explain the development of allergic reactions. Our investigations confirm that ASNase is degraded by AEP. In addition, ASNase, as well as Erwinase, is inactivated by a second lysosomal cysteine protease, cathepsin B (CTSB). Our results identify what we believe is a novel mechanism of drug resistance in childhood ALL mediated by lysosomal cysteine proteases present in lymphoblasts.

\section{Results}

$A E P$ and CTSB present in pre-B lymphoblasts degrade ASNase. AEP expression of 3 ALL (SD1, REH, SupB15) and 1 B-lymphoblastoid (HRC57) cell line was examined by quantitative PCR and Western blotting. SD1 and HRC57 but not REH and SupB15 showed high levels of AEP expression (Supplemental Figure 1A). To determine whether proteases in cellular lysates degraded ASNase, cell lysates were incubated with the drug. A distinct smaller band was observed on probing in cell lysates, confirming cleavage of ASNase

\section{Figure 1}

ASNase is degraded by cysteine proteases present in pre-B lymphoblasts. Immunoblotting for ASNase following incubation of ASNase with whole-cell lysates from cell lines. (A) ASNase is cleaved by cellular lysates of REH, SD1, and HRC57. (B) SD1 lysates were preincubated with various protease inhibitors prior to incubation with ASNase. ASNase cleavage is partially prevented by AEPi and by a PIC. Complete inhibition was achieved with a combination of AEPi and PIC. Combining either E64 or leupeptin, both components of PIC, with AEPi also prevents degradation. E64 and leupeptin both inhibit CTSB, and a combination of AEPi and CTSBi blocks cleavage of ASNase by SD1 cell lysate. Vertical line demarcates different gels; dashed vertical line indicates noncontiguous lanes within a gel. (C) CTSBi alone prevents ASNase cleavage by REH cell lysate.

(Figure 1A). In SD1 cell lysates, this cleavage was only partially prevented by the AEP-specific inhibitor MV026630 (AEPi) (23) (Figure 1B). Complete inhibition was achieved when AEPi was combined with a protease inhibitor cocktail (PIC) or either of its 2 individual components, E64 and leupeptin, both cysteine protease inhibitors (Figure 1B and Supplemental Figure 1B). E64 and leupeptin both inhibit CTSB. ASNase degradation was completely blocked when SD1 lysates were preincubated with a combination of AEPi and the CTSB-specific inhibitor CA074Me (CTSBi) (24) (Figure 1B). In REH cells, which do not express AEP, CTSBi by itself inhibited degradation (Figure $1 \mathrm{C}$ and Supplemental Figure $1 \mathrm{C}$ ). Thus, ASNase is degraded by CTSB alone (REH) or by a combination of CTSB and AEP (SD1) (Supplemental Table 2). As shown in Figure 2A, from our previously published gene expression dataset $(20,25)$, we determined that CTSB is constitutively expressed at low levels by all leukemic cells and upregulated in acute myeloid leukemia (AML) $(P=0.0002)$. AEP was primarily overexpressed in high-risk cytogenetic subgroups of pre-B ALL $(P<0.0001)$. The expressions of these 2 proteases in leukemic cells obtained from patients with ALL are shown in Figure 2B (Supplemental Figure 2 and Supplemental Table 3). Lysates from these leukemic blast cells also degraded ASNase (Supplemental Figure 3), either through CTSB alone or through a combination of CTSB and AEP; examples from 4 patients are shown in Figure 2C.

AEP degrades ASNase but not Erwinase. To determine the specificity of cleavage of asparaginase by the 2 lysosomal proteases, we incubated purified recombinant AEP and CTSB with various commercial preparations of asparaginases. As shown in Figure 3, purified CTSB cleaved both Erwinase and all forms of commercially available ASNase. AEP specifically cleaved ASNase only. AEP is expressed by renal, splenic, and hepatic tissue and dendritic cells but not normal white cells (26) or precursor B cells (27). It is therefore aberrantly expressed by pre-B lymphoblasts. AEP expression was predominantly seen in high-risk pre-B ALL patients. These are the patients who also show low asparaginase activity and/or develop hypersensitivity. Our results thus suggested that AEP modulates the therapeutic response to ASNase. We therefore further investigated the mechanism of AEP-mediated degradation of ASNase.

Modeling AEP cleavage of ASNase. To determine the AEP cleavage sites of ASNase, we peptide-sequenced the digested frag- 
A

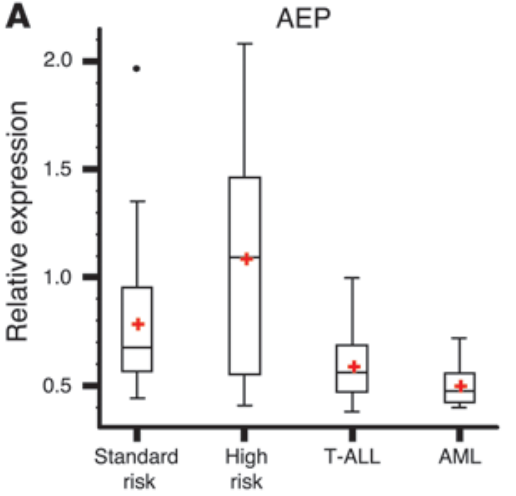

B

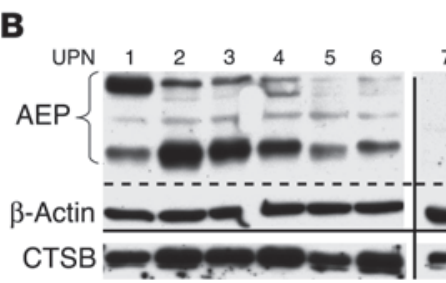

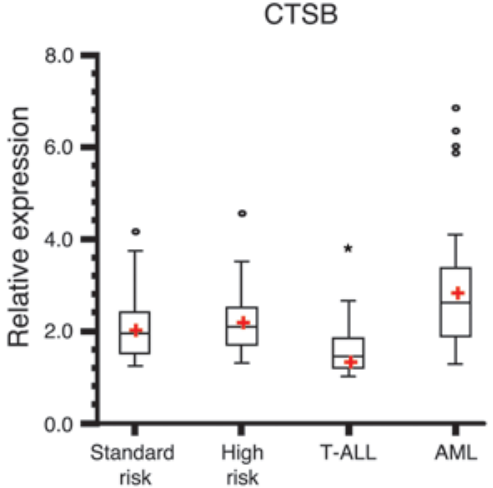

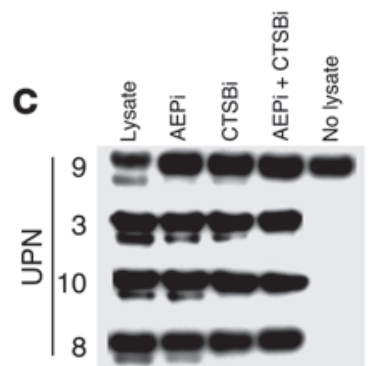

Figure 2

$A E P$ is relatively overexpressed in cytogenetic high-risk subsets of pre-B ALL, while CTSB expression is relatively uniform across ALL subtypes (A) Box-plot representations of relative AEP and CTSB microarray expression in leukemic blasts. Categories of acute leukemia are shown on the $x$ axis. High risk $(n=24)$ refers to pre-B ALL subtypes with high-risk cytogenetic features, including BCR-ABL1, MLL rearrangements, iAMP21, and hypodiploidy. The other categories include standard risk pre-B ALL $(n=59)$, T cell-lineage ALL $(n=11)$, and AML $(n=26)$. The $y$ axis represents the relative gene expression level of either AEP or CTSB. Box plots for each category of ALL represent the interquartile range of values, the whiskers represent the smallest and largest values for each category, and the horizontal lines and plus symbols denote the median and mean respectively; outlier values are represented by circles and asterisks. (B) Primary lymphoblasts express CTSB and AEP. Immunoblots of primary ALL samples (UPN, unique patient number) and cell lines. Precursor ( $56 \mathrm{kDa})$ and active ( $36 \mathrm{kDa})$ forms of AEP are detected. The 20 and 25 $\mathrm{kDa}$ bands in the CTSB blot represent the heavy chains of mature CTSB dimers; $\beta$-actin was the loading control. Lines demarcate different gels; dashed lines denote spliced noncontiguous lanes within gels. (C) Lysates of primary blast cells cleave ASNase. Cleavage is inhibited by CTSBi in patients 8 and 10 and by a combination of CTSBi and AEPi in patients 3 and 9. A no-lysate control is shown for patient 9 only.

ments. Sequence analyses identified 3 typical AEP cleavage sites in ASNase, N24, D124, and N143 (Figure 4A). The cleavage products retain intact known B cell epitopes of ASNase (28), suggesting that AEP-induced cleavage could accelerate antigen presentation (29) of ASNase, leading to hypersensitivity and/or development of antibodies. The effects of proteolytic cleavage were modeled using the published crystal structure of ASNase (30). As illustrated in Figure $4 \mathrm{~B}$, ASNase is active as a tetramer (30). Structural analyses indicate that N24 is located on the surface of the tetramer, proximal to the functional site. Residue D124 directly stabilizes the catalytic site, forming 2 hydrogen bonds to the backbone atoms of residues M115 and R116, which are part of a loop within the active site (Figure 4C). Residue N143 is located on the surface of the tetramer and is neither close to the active site nor close to the monomermonomer interface. Thus, the model suggests that cleavage at or replacement of N24 and D124 but not N143 would affect tetramerization and/or catalysis. Further support for this model comes from the comparison of the amino acid sequences of ASNase and Erwinase (Figure 5A). The 2 proteins have a sequence identity of $46.5 \%$. The region around N24 is not highly conserved, and G is found in this position in Erwinase. D124 and neighboring amino acids are highly conserved. While the region around N143 is also conserved, N143 itself is replaced by G in Erwinase.

The relative accessibility of the N24 and N143 cleavage sites, taken together with the observation that Erwinase is not cleaved by
AEP, strongly suggests that AEP initially cleaves ASNase at either $\mathrm{N} 24$ or N143. N24 is a member of a loop responsible for opening and closing the binding pocket (31). Figure 5B shows the structure of the loop with the open and closed conformations superimposed. In the open conformation, the $\mathrm{N} 24$ residue was exposed on top of an $\alpha$-helix and projects out to the solvent while in the closed conformation; this residue lies in a grove formed by the monomermonomer interface. Therefore, this residue will be more prone to cleavage when the loop is in the open conformation. To further determine which of the 2 residues represents the primary cleavage site, we estimated the accessibility of both sites for AEP. As there is no crystal structure of AEP, we performed molecular modeling studies (3D-JIGSAW) (32), which predicted that the active site of AEP lies in a deep pocket of an average radius of approximately $7 \AA$. Figure 5C shows the surface accessibility of ASNase drawn with a probe radius of $7 \AA$. N24 in the open loop conformation clearly lies on a convex surface patch that extends into the environment. Consequently, it is easily accessible to AEP. In contrast, N143 is placed inside a groove, which makes it difficult for AEP to access. This suggests that N24 is the primary AEP cleavage site in the ASNase molecule. Cleavage at this site could destabilize the tetramer, permitting AEP access to other cleavage sites, such as D124. Thus, modifying $\mathrm{N} 24$ could create an ASNase molecule resistant to AEP cleavage.

ASNase N24G mutant resists AEP cleavage but has reduced enzymatic activity. To verify the molecular modeling predictions, we synthe- 

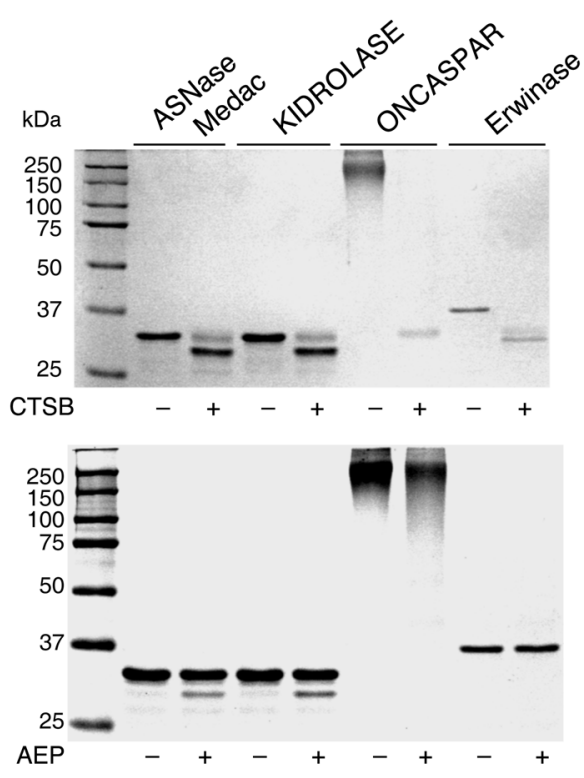

sized N24G, D124G, N143G, N24G/D124G, and D124G/N143G recombinant mutant asparaginases. Substitution with $\mathrm{G}$ at N24 and N143 residues was based on the Erwinase sequence (Figure 5A). As shown in Figure 6 (also Supplemental Figure 4), all 3 D124 mutants were completely degraded by purified AEP and almost so by the cellular lysates. The N24G recombinant with the conserved D124 residue was the only mutant resistant to AEP cleavage. This confirms that AEP first cleaves native ASNase at N24. Cleavage at

\section{Figure 3}

CTSB degrades both ASNase and Erwinase, while AEP is specific for ASNase. Coomassie blue-stained SDS-PAGE images of asparaginases $(4 \mu \mathrm{g})$ incubated with $(+)$ or without $(-)$ human recombinant CTSB $(0.5 \mu \mathrm{g})$ (upper panel) or AEP $(0.4 \mu \mathrm{g})$ (lower panel). KIDROLASE and ASNase Medac are commercially available ASNase preparations and are detected as monomeric compounds. ONCASPAR is a polyethylene glycol-conjugated version of ASNase Medac ASNase and is detected as a high-molecular weight complex.

this site is predicted to destabilize the tetramer and presumably allows AEP access to D124 and N143.

We next examined the enzymatic activity of the various mutant ASNase compounds (Table 1). Substituting N24 with G based on the Erwinase sequence resulted in a mutant that was significantly less active when compared with the WT recombinant (relative activity $45 \%$ ). The D124G mutants showed minimal activity. This confirms the prediction made by protein modeling, i.e., D124 is critical to the functional structure of ASNase. Equally, N143 is not a critical cleavage site, since the N143G mutant was similar to the WT recombinant, both in terms of activity and susceptibility to AEP cleavage.

Thus, activity of ASNase appears to depend not only on D124 but also on N24. Further molecular dynamic (MD) simulations unraveled an important enzymatic function for N24. Close investigation of the MD trajectory revealed the formation of an interchain hydrogen bond in the tetramer that contributes to the stabilization of the monomer-monomer interface (Figure 7A). In contrast to the native conformation, this interchain bond was not formed in the N24G mutant (Figure 7B). This mutation creates a

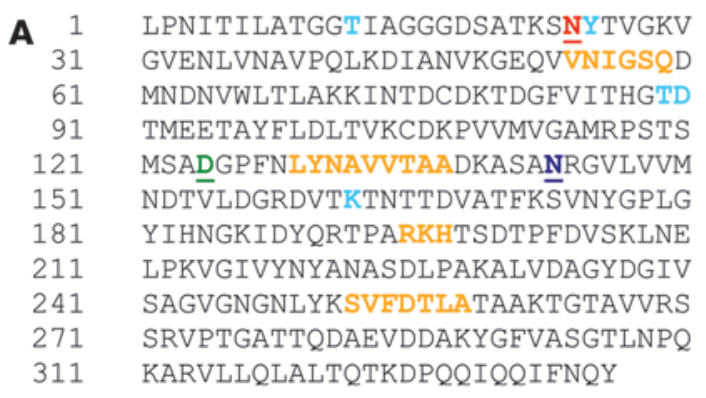

30
60
90
120
150
180
210
240
270
300
327

C

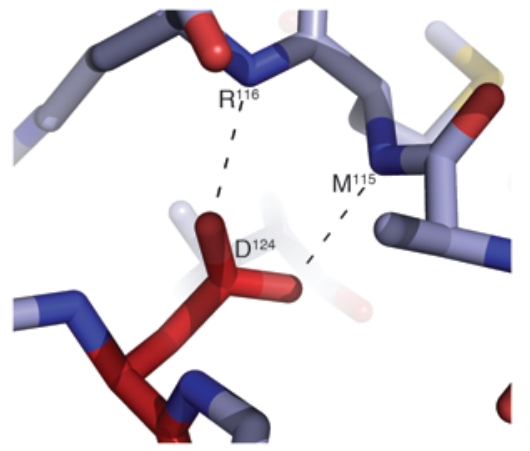

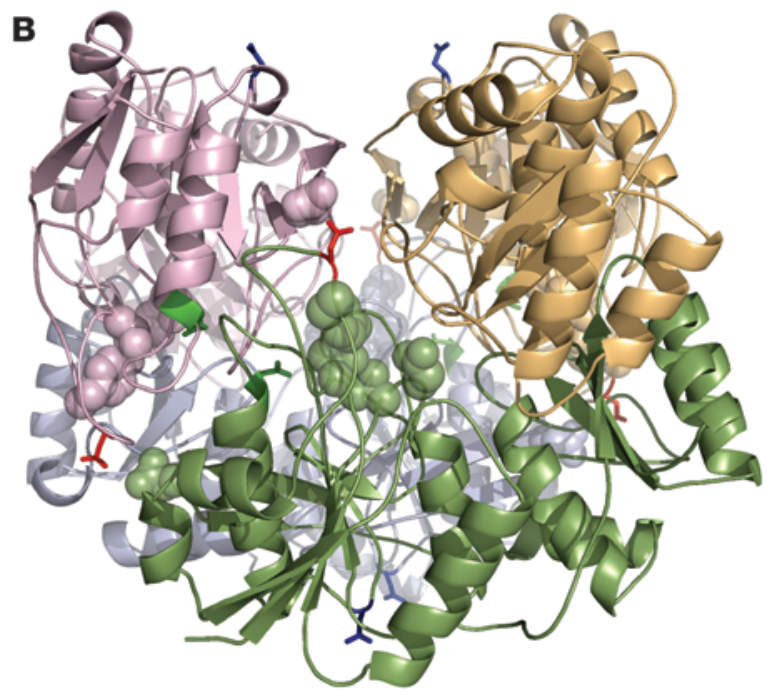

Figure 4

Protein modeling of AEP cleavage sites of ASNase. (A) Amino acid sequence of Medac ASNase showing AEP cleavage sites (underlined) as identified by N terminus Edman sequencing. N24 residue is shown in red, D124 in green, N143 in dark blue. Previously described potential antigenic sequences are shown in orange and catalytic residues in light blue. (B) Asparaginase functions as a tetramer, and each monomer is shown in a separate color. The active site is shown in spacefill representation. N24 is highlighted in red, D124 in green, and N143 in dark blue. (C) The hydrogen bond network formed by D124 with the neighboring amino acid residues M115 and R116 helps directly stabilize the ASNase catalytic site. 


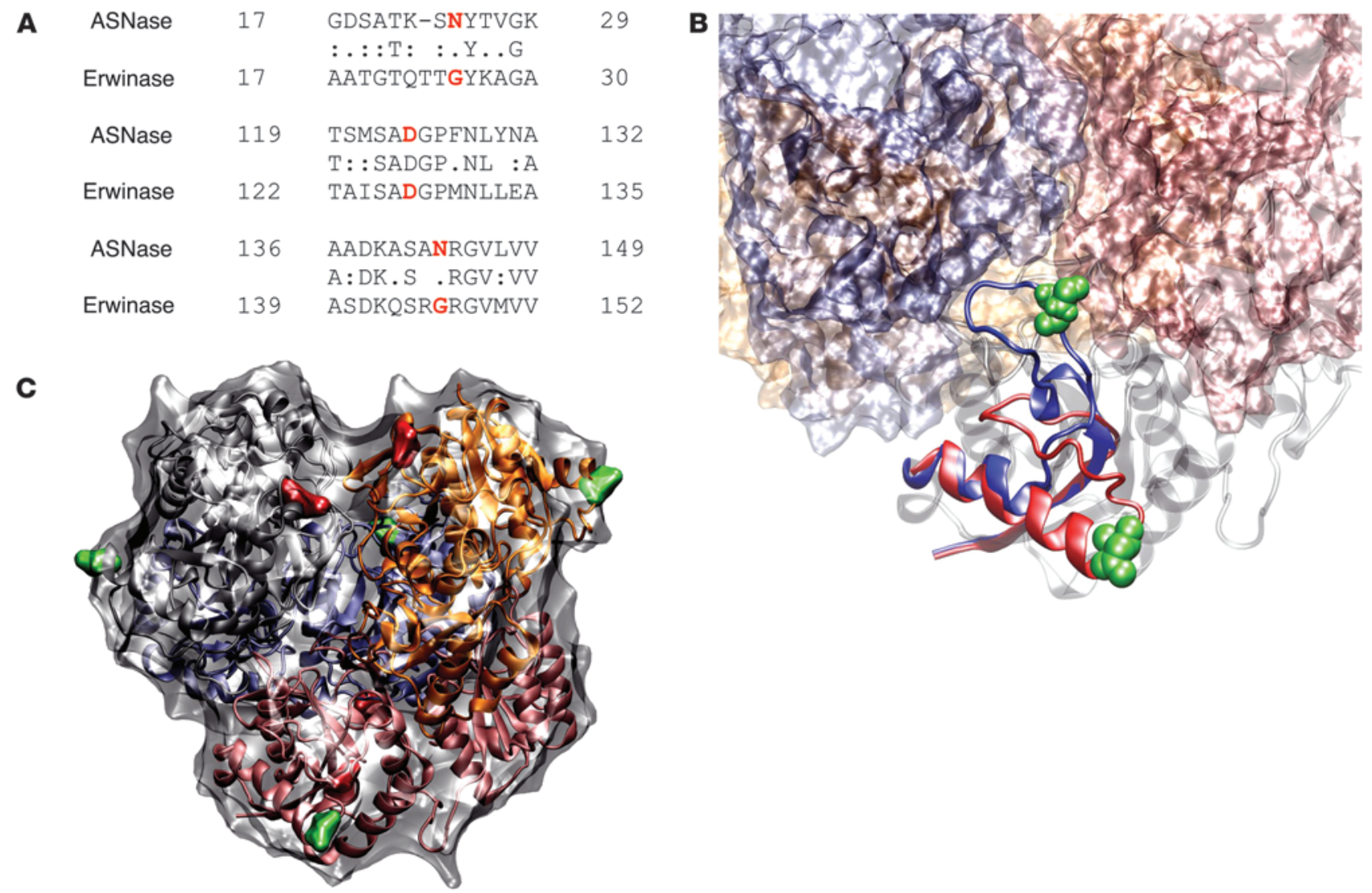

\section{Figure 5}

Molecular modeling suggests that AEP first cleaves at the N24 residue. (A) Pairwise protein alignment for ASNase and Erwinase. Top row, ASNase; bottom row, Erwinase; middle row, consensus. Conserved amino acids are listed, favorable mismatches are shown as a colon, neutral mismatches as a period, and unfavorable mismatches as a space. AEP-cleaved amino acids in ASNase and the corresponding amino acids in Erwinase are shown in red. (B) The flexible loop containing N24. The open conformation is depicted in red, the closed conformation in blue, N24 in green. (C) Representation of the solvent accessibility surface of the ASNase tetramer generated with a 7-Å probe radius. N24 is highlighted in green; N143 is highlighted in red. Monomers are shown in different colors. The location of N24 on an exposed convex surface patch renders it readily accessible to AEP cleavage.

knockon effect, disrupting a hydrogen bond network close to the active site. As a result, the downstream Y25 changes its conformation. This may affect the loop responsible for opening and closing the binding pocket (residues 24 and 25 both form part of this loop). Furthermore, Y25 has been reported to be crucial for the correct placement and stabilization of T12 (31), another member of the active site (33). In the native molecule, the hydroxyl group of 12 points into the substrate-binding pocket and is responsible for nucleophilic attack on the substrate. To investigate the position of the T12 hydroxyl group in the course of the MD simulation, we monitored the side chain torsion angle, $\chi 1$, T12. The binary state of the angle, which orients either the hydroxyl or the methyl group of T12 toward the active site, is shown for both the native protein (Figure 7C) and the N24G mutant (Figure 7D). The appropriate native orientation of the $\mathrm{T} 12$ hydroxyl group toward the substrate-binding pocket was maintained during the course of MD simulation for the WT molecule. However, MD simulation in the N24G mutant showed that the methyl group of T12 occupied the substrate-binding pocket for $34 \%$ of the simulation time. Thus, T12 in the N24G mutant is unable to correctly interact with the substrate. Nevertheless, this rotation of the T12 side chain is reversible in the N24G mutant, explaining the reduced but not complete loss of functionality. Erwinase has $\mathrm{G}$ in position 24 and is less active when compared with ASNase. MD simulations for Erwinase revealed that, although the T12 orientation is similar for both proteins, the active-site loop is more flexible in Erwinase (data not shown). This may be responsible for its lower activity. For efficient catalysis, the active-site loop needs to close upon the substrate molecule bound in the active site. It is probable that the more flexible active loop in Erwinase also makes it less able to close tightly on the substrate molecule, which may partly explain Erwinase's reduced catalytic activity. However, it should be noted that there are also significant differences in the neighborhood of the active site between Erwinase and ASNase (overall sequence identity 47\%; the active-site loop in Erwinase is 1 residue longer than in ASNase), and, consequently, all direct comparisons of structure-activity relationships between the 2 asparaginases may only be qualitative.

Active AEP is expressed in a peripheral lysosomal compartment in lymphoblasts. To better understand the possible interactions of AEP with ASNase, we investigated the location of active AEP in SD1 cells. Labeling using an activity-based probe (AEP-ABP) (34) con- 


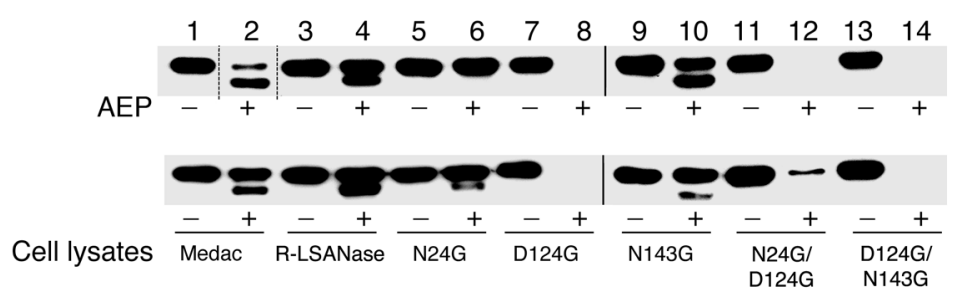

\section{Figure 6}

An N24G mutant resists AEP cleavage. Immunoblots of commercial (Medac) and recombinant WT (R-LASNase) and mutant ASNase proteins incubated with (+) and without (-) recombinant AEP $(0.4 \mu \mathrm{g})$ or whole-cell lysates (HRC57). N24G is resistant to AEP cleavage. D124 mutants are degraded completely by AEP and cellular lysates. Vertical lines demarcate different gels; dashed vertical lines denote spliced noncontiguous lanes within gels. firmed that AEP is expressed by SD1 cells but not by REH and SupB15 (Supplemental Figure 5A). There is considerable interand intracellular heterogeneity in the pattern of AEP staining in SD1, with both punctate and aggregate staining patterns observed (Supplemental Figure 5, B and C). As shown in Figure 8A, AEP colocalizes with the acidotropic LysoTracker Red probe, presumably to mature lysosomes. Thus, our results confirm a previous observation that precursor AEP and the mature active protein are localized in distinct intracellular endosomal/lysosomal and acidic vesicular compartments (35). However, contrary to the results of the earlier report, in which active AEP was found to be predominantly perinuclear, active AEP in SD1 cells localized at the periphery of the cell in well-circumscribed lysosome-associated membrane protein 1-positive (LAMP1-positive) compartments (Figure 8B and Supplemental Figure 5D). This suggests that either cell lysis or exocytosis could expose ASNase to active AEP, resulting in ASNase degradation.

\section{Discussion}

Among the lysosomal proteases, AEP is peculiar in its strict specificity and selectivity in cleaving after asparagine and aspartate. We have utilized this to accurately dissect the sequence of AEP cleavage of ASNase and identify key residues. The predictive accuracy of the modeling allowed us to create an ASNase resistant to AEP cleavage and establish the critical nature of N24 and D124. CTSB does not show similar substrate specificity. It too cleaves at N24, but substitution at this residue does not prevent CTSB cleavage (N. Patel, unpublished observations). Thus the open conformation of the loop containing N24 exposes the molecule to proteolytic attack by both cysteine proteases. Indeed, the shorter half-life of Erwinase may be due to the increased flexibility of this loop and/or the activity of other proteases. CTSB is classified as a housekeeping gene and is constitutively expressed in all tissues (36); unlike AEP, its expression in lymphoblasts may reflect basal activity. CTSB is exocytosed or secreted by the cell into extracellular fluid (37). Our results show that active AEP lies in a well-defined compartment at the periphery of the blast cell and suggest that it too may undergo a similar process. Indeed, this property has been exploited to create an AEP-activated cellimpermeable doxorubicin molecule that showed high selectivity for AEP-producing tumor cells (38). Thus, active proteases would be expected to interact with asparaginase as a substrate.

The mechanism of clearance of L-asparaginase has not been determined. While CTSB is expressed ubiquitously, AEP expression is restricted primarily to renal tissue. It is tempting to speculate that CTSB is involved in the normal degradation and clearance of both ASNase and Erwinase, although a clearance role for other proteases cannot be excluded. In the case of ASNase, clearance at first exposure may be accelerated by AEP-expressing leukemic cells, contributing to the slow early response to therapy. AEP degradation may additionally promote sensitization, resulting in enhanced ASNase clearance on subsequent exposures. Though the levels of expression of these proteases are likely to significantly modulate asparaginase levels, other mechanisms also contribute to drug resistance. For example, $\mathrm{T}$ cell lymphoblasts are relatively resistant to asparaginase (7). Neither protease is expressed at high levels in this subgroup, and thus for T cell disease, the mechanism of ASNase resistance remains unknown.

Both CTSB and AEP have been implicated in antigen processing and presentation, cleaving of membrane-bound proteins, cardiovascular disease, and matrix degradative processes in cancer. AEP not only inactivates ASNase, but the cleaved peptides could potentiate antigen presentation. Such a process provides a unified explanation as to why some children, primarily those in the high-risk group, do not show adequate response to and/or develop allergic reactions to ASNase. We have not demonstrated that AEP cleavage potentiates the allergic response to ASNase. These experiments are difficult to do retrospectively in patients who have completed therapy for childhood ALL. We are testing the validity of these observations in the context of a prospective clinical trial designed to evaluate whether AEP expression, within the setting of multidrug therapy, correlates with decreased asparaginase activity, increased antibody formation, and slower response to therapy. If indeed such an association can be made, a number of possibilities exist to improve outcome and decrease morbidity in children with ALL undergoing chemotherapy. Patients can be screened for AEP expression at diagnosis. Those showing high levels can be offered Erwinase instead of ASNase. This will allow optimal asparagine depletion without the early development of hypersensitivity. Though the AEP-resistant N24G mutant is not as active as the WT, further modeling and engineering of ASNase is ongoing and likely to produce a highly active enzyme that is cleavage resistant. In the future, such a synthetic product could replace current bacterial products. Another option would be to use specific protease

\section{Table 1}

Enzymatic activity of the different recombinant ASNase products

$\begin{array}{lcc}\text { Asparaginase } & \text { IU/mg } & \text { Ratio }^{\mathbf{A}} \\ \text { WT ASNase } & 1012 & 1 \\ \text { N24G ASNase } & 453 & 0.45 \\ \text { D124G ASNase } & 28 & 0.03 \\ \text { N143G ASNase } & 1106 & 1.09 \\ \text { N24G/D124G ASNase } & 36 & 0.04 \\ \text { D124G/N143G ASNase } & 53 & 0.05\end{array}$

Measurement of enzymatic activity of the different recombinant ASNase products using the Medac Asparaginase-Activity-Test (MAAT) assay kit (Medac). ARecombinant ASNase activity/WT ASNase activity. 
A
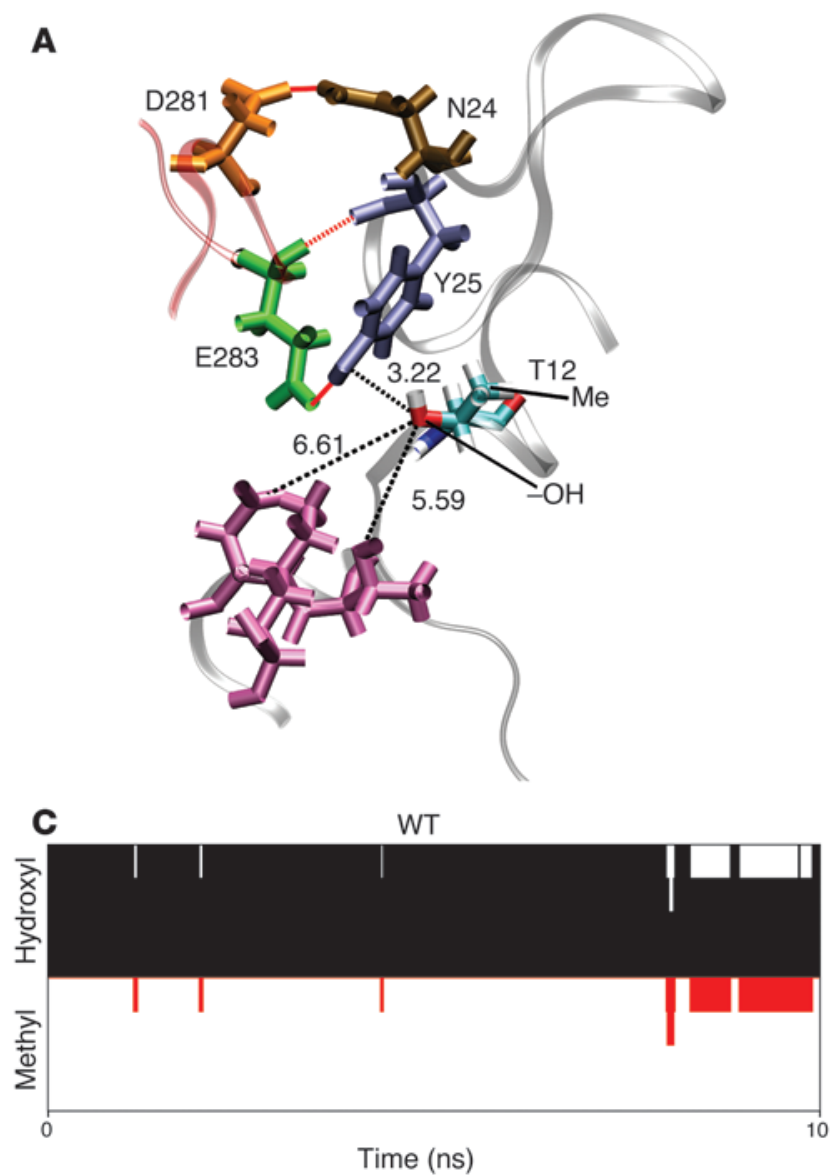

B
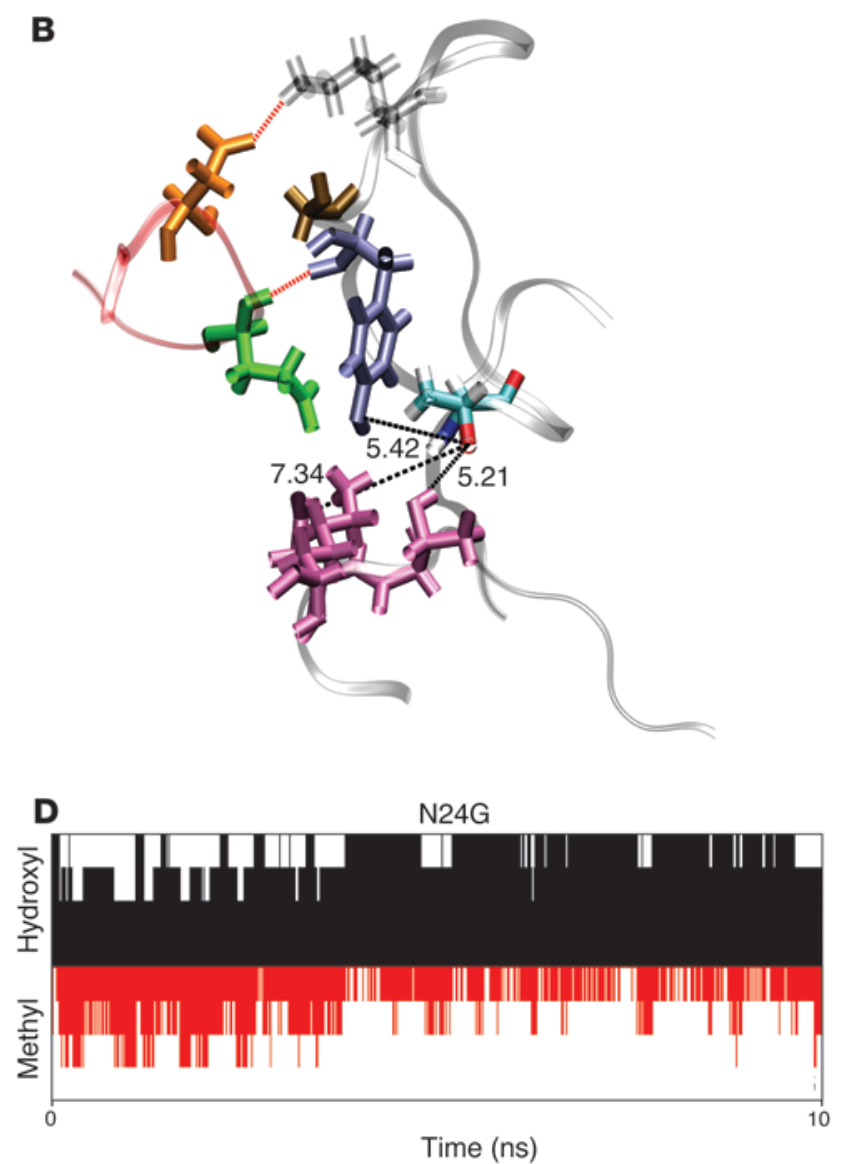

\section{Figure 7}

Decreased enzymatic activity of the N24G mutant is explained by MD simulations of N24 interactions. The hydrogen bond network around N24 in WT ASNase (A) and the N24G mutant (B) is shown. Residue T12 is colored according to atom types. Key distances (in $\AA$ ) show position of the T12 hydroxyl group with respect to the active site. Residues N24 and Y25 are colored brown and blue, respectively; other residues of the active site are in purple. Two residues that belong to another monomer but form hydrogen bonds (red) with either N24 or Y25 are shown in orange (D281) and green (E283). MD simulation showing orientation of the T12 hydroxyl group with respect to all 4 active sites at each time point during simulation in native ASNase (C) and the N24G mutant (D). The black part of each graph represents the fraction when the T12 hydroxyl group is in correct orientation with respect to the 4 active sites. The red part of each graph represents the fraction when the T12 hydroxyl group is incorrectly oriented relative to the 4 active sites (methyl instead of the hydroxyl group pointing toward the center of the active site).

inhibitors in conjunction with ASNase therapy. AEPi, used in this study, though cell permeable, is irreversible. For therapeutic uses, a reversible inhibitor is desirable.

The current therapeutic focus in childhood ALL is on identifying drugs that target the specific genetic defects in leukemic cells that lead to therapeutic failure (39). How these novel agents will be introduced remains unclear, as present strategies for treating children with ALL are highly successful. Thus, optimization of existing drugs continues to remain a practical alternative. Indeed, in the last decade, improvements in outcome in the United Kingdom reflect the better use of existing agents $(40,41)$. While dose-limiting toxicities for many of the drugs used in ALL therapy have been reached, this is not the case for asparaginases. Our studies identify a putative novel mechanism of drug resistance to the key antileukemic agent ASNase and present insights into the variations in response to ASNase in childhood ALL. This provides a platform for further optimization of asparaginase therapy with the potential of decreasing morbidity and improving outcome.

\section{Methods}

Cell lines. HRC57, SD1, and REH cell lines were obtained from Cancer Research UK Central Cell Service Department. The SupB15 cell line was obtained from DSMZ (German Collection of Microorganisms and Cell Cultures). All cell lines were cultured at $37^{\circ} \mathrm{C} / 5 \% \mathrm{CO}_{2}$ in RPMI 1640 GlutaMAX (Invitrogen) with 10\% FBS (SLI).

Primary human leukemia samples. Excess bone marrow aspirate samples were obtained at diagnosis from children with acute leukemia. Mononuclear cells were isolated from marrow samples by density centrifugation (Lymphoprep; Axis-Shield) and cryopreserved in RPMI 1640 with 20\% FBS and $10 \%$ DMSO. Samples were only processed from patients who were treated on national protocols and who had consented to the storage and use of excess material for ethically approved research. The project was approved by the Tameside and Glossop Local Research Ethics Committee, Manchester, United Kingdom.

Gene expression analyses. Gene expression on diagnostic cells from $94 \mathrm{ALL}$ and 26 AML patients was analyzed on Affymetrix HG-U133A oligonucleotide arrays using the GeneSpring 7.3.1 platform (Agilent Technologies) 
A
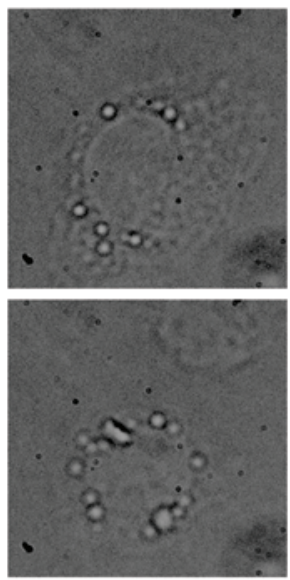

B
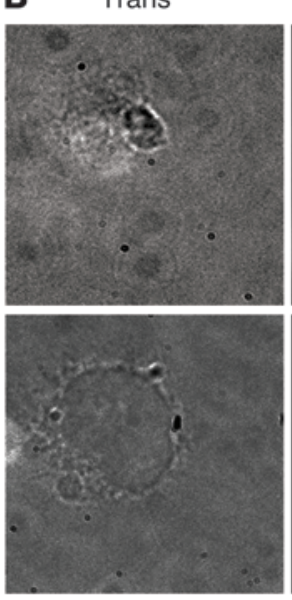

DAPI
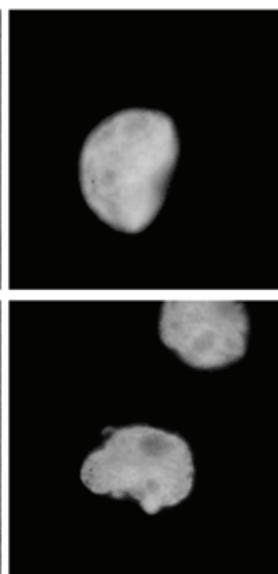

DAPI
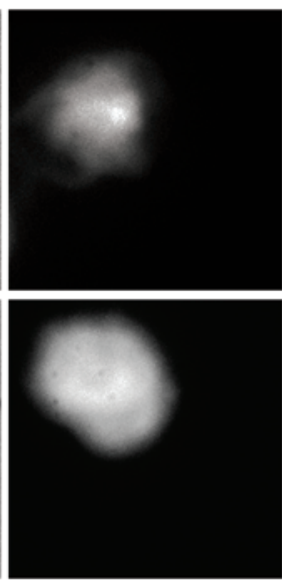

LyTR
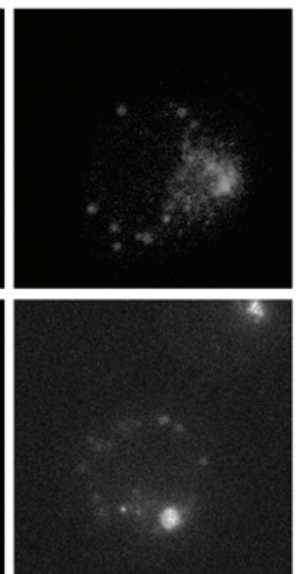

AEP-ABP
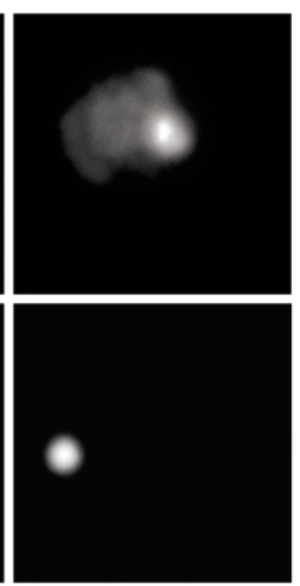

AEP
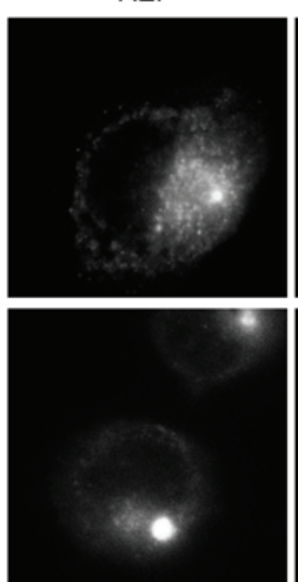

LAMP1
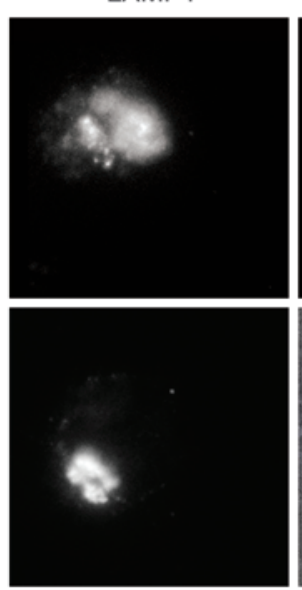
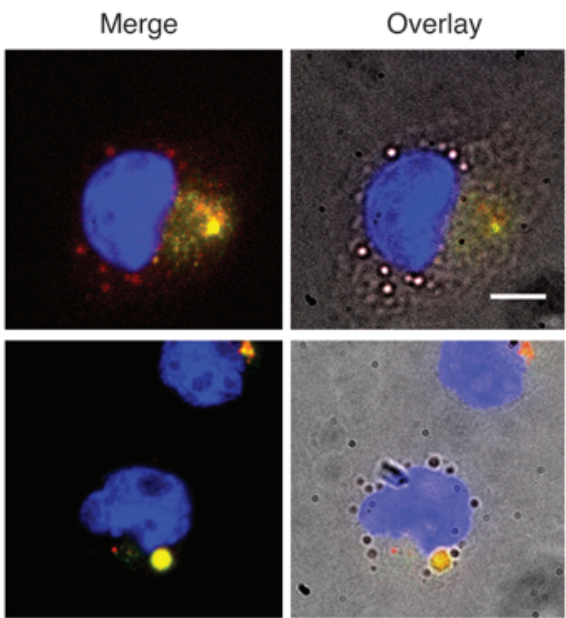

Figure 8

Mature active AEP in SD1 cells accumulates toward the cell periphery in discrete acidic LAMP1-positive compartments. (A) Immunostaining localizes AEP in peripheral acidic compartments labeled by an acidotropic lysosomal probe. AEP immunostaining in formalin-fixed cytospins of SD1 cells preincubated with the cell-permeable LysoTracker Red DND-99 lysosomal probe (LyTR). The large yellow aggregate fluorescence in the images represents overlay of green (AEP) and red (lysosomal probe) fluorescence. Original magnification, $\times 100$, optivar magnification, 1.6. (B) Live-cell labeling AEP-ABP localizes active AEP in peripheral LAMP1-positive vesicles. LAMP1 immunostaining in formalin-fixed cytospins of SD1 cells preincubated with AEP-ABP. Images indicate active AEP (red) at the cell periphery, localized in what appears to be large globular vesicles ringed by LAMP1 (green). Original magnification, $\times 100$, Optivar 1.6. DAPI (blue) stains the nucleus; Trans refers to brightfield images. Scale bars: $5 \mu$ m.

as described previously $(20,25)$. Results were validated by quantitative PCR (Supplemental Methods).

Degradation of ASNase. Washed cell pellets from cell lines and primary samples were lysed by freeze-thawing in digestion buffer $(50 \mathrm{mM}$ trisodium citrate buffer, $\mathrm{pH} 4.5,5 \mathrm{mM}$ DTT). Lysates were clarified by centrifugation and the supernatants stored at $-80^{\circ} \mathrm{C}$. For incubation experiments, 2 to $4 \mu \mathrm{g}$ of ASNase (Medac) was incubated at $37^{\circ} \mathrm{C}$ with whole-cell lysates $(20 \mu \mathrm{g})$ overnight or for 3 hours with either activated recombinant human AEP (kindly provided by Colin Watts, University of Dundee, Dundee, United Kingdom) or CTSB (R\&D Systems). Samples were immunoblotted as below. Additional degradation experiments were performed using Erwinase (Opi Pharma) and alternative preparations of ASNase (KIDROLASE, OPI Pharma; ONCASPAR, Medac GmbH). For identification of AEP cleavage sites, the ASNase digest was resolved by SDS-PAGE and transferred to a PVDF membrane. Bands were then excised and submitted for N-terminal amino acid sequencing by Edman degradation (Aberdeen Proteomics).
Immunoblotting. Total cellular proteins were extracted using $0.1 \% \mathrm{NP}-40$ lysis buffer and quantified using a modified Lowry assay (Bio-Rad). Lysates were placed in $5 \times$ sample loading buffer and resolved using $10 \%$ SDSPAGE. Following semidry transfer, PVDF membranes were probed with the appropriate primary antibodies. The following primary antibodies were used: rabbit polyclonal anti-ASNase (1:5000; Abcam), goat polyclonal antihuman AEP (1:2500; R\&D Systems), rabbit polyclonal anti-human CTSB (1:5000; BIOMOL International), and monoclonal $\beta$-actin (murine clone AC-15, 1:5000; Sigma-Aldrich). HRP-conjugated secondary antibodies (goat anti-rabbit, goat anti-mouse from Pierce, Thermo Scientific; donkey anti-goat from Santa Cruz Biotechnology Inc.) and the enhanced chemiluminescence reaction (Pierce; Thermo Scientific) were used for detection.

Inbibition of ASNase cleavage. Whole-cell lysates $(20 \mu \mathrm{g})$ in digestion buffer were preincubated for 15 minutes at $37^{\circ} \mathrm{C}$ with protease inhibitors either alone or in combination. ASNase $(2-4 \mu \mathrm{g})$ was added to the reaction and then incubated overnight at $37^{\circ} \mathrm{C}$. Protease inhibitors included the PIC P8340 
CTSBi $(1 \mu \mathrm{M})$, E64 $(0.14 \mu \mathrm{M})$ (all from Sigma-Aldrich), leupeptin $(0.2 \mu \mathrm{M}$; Roche), and AEPi $(10 \mu \mathrm{M})$. P8340 includes serine, cysteine, thiol, aminopeptidase, and aspartyl protease inhibitors but does not include metal chelators.

Sequence alignment and molecular modeling. Protein sequences were obtained from the Entrez Protein database (accession numbers P00805 [ASNase] and P06608 [Erwinase]). Lipman-Pearson pairwise protein alignment for ASNase and Erwinase was performed using MegAlign (DNASTAR) using a k-tuple of 2, gap penalty of 4 , and gap-length penalty of 12 . The crystal structure of ASNase was obtained from the Protein Data Bank (ref. 42; PDB entry 3ECA). All 3 cleavage sites were investigated using this crystal structure. A structural analysis was performed to reveal the functional context of each cleavage site. The catalytic residues were extracted from the Catalytic Site Atlas Internet database (33). The proximity and interactions of ASNase catalytic sites with each of 3 AEP cleavage residues (N24, D124, N143) were analyzed. Prior to each MD simulation, all bound substrate molecules (aspartic acid) were removed. Crystal water molecules were retained for ASNase, as they are required for correct functioning of the active site. Asparagine was substituted by glycine in the N24G mutant. Each protein was immersed in a rectangular box of water, and $\mathrm{Na}^{+}$and $\mathrm{Cl}^{-}$ions were added to obtain the salt concentration of $0.2 \mathrm{M}$. Each system was subsequently minimized and equilibrated for 50 ps in NVT (fixed particle number at constant volume and temperature) and NPT (fixed particle number at constant pressure and temperature) ensembles with backbone atoms restrained to their original positions. After the equilibration, each system was simulated for $10 \mathrm{~ns}$ at room temperature (298K). All MD simulations and analyses were performed with the AMBER suite (43).

Creation of ASNase mutants, protein expression, and purification. The full-length coding sequence of ASNase in PPT002 vector (AnsB) in PPTOT2 vector (Medac) was $X h o I / E c o R I$ digested, ligated into the pRSETB vector (Invitrogen), and transformed into competent cells. Plasmid DNA from transformed cells was used to generate a series of mutants using site-directed mutagenesis (Stratagene) and the following primers: P1: 5'-GACTCCGCAACCAAATCTGGCTACACAGTGGGTAAAG-3' for N24G; P2: 5'-ACGTCTATGAGCGCAGGCGGTCCATTCAACCTGT-3' for D124G; and P3: 5'-TAAAGCCTCCGCCGGTCGTGGCGTGCTG-3' for N143G. Combinations of P1 plus P2 and P2 plus P3 were used to create the N24G/D124G and D124G/N143G mutants, respectively. All constructs were verified by automated DNA sequencing. For protein expression, E. coli BL21(DE3)pLysS competent cells were used. Single clones were cultured to an $\mathrm{OD}_{600}$ of $0.4-0.6$, and isopropyl $\beta$-D-thiogalactopyranoside (MP Biomedicals) was added to induce protein expression. After an additional 4 hours, cells were harvested by centrifugation and recombinant protein was extracted from the supernatant by osmotic lysis in ice-cold water. Further purification of recombinant protein was performed by Wacker Chemie AG.

Estimation of enzymatic activity. Enzymatic activity of native and mutant ASNases was estimated using the quantitative Medac Asparaginase-Activity-Test (MAAT) assay kit as described previously (44).
Microscopy. SD1 cells were labeled with cell-permeable fluorescent probes for active AEP (AEP-ABP, $1 \mu \mathrm{M}$ at $37^{\circ} \mathrm{C}$ for 2 hours) and mature endolysosomes $\left(100 \mathrm{nM}\right.$ at $37^{\circ} \mathrm{C}$ for 45 minutes; LysoTracker Red DND-99, Molecular Probes, Invitrogen). Labeled cells were subsequently fixed (10\% neutral buffered formalin), permeabilized (0.25\% Triton X-100 in PBS), and immunostained for either LAMP1 (1:100; BD Biosciences) or for AEP (1:100; R\&D Systems), using standard methods. Images were acquired on a Zeiss Axiovert 7D microscope using a cooled electron-multiplying chargecoupled device (EMCCD) low-light camera (Photometrics Cascade II:1024 megapixel) operated by the MetaMorph FRAP Advanced Imaging software (version 7.5.2.0; Molecular Devices). Acquired images were processed using Adobe Photoshop CS3 software (Adobe Systems). Full details are provided in Supplemental Methods.

Statistics. One-way ANOVA was used to examine differences in mean expression of AEP and CTSB among subgroups of leukemia (version 15.0; SPSS). $P<0.05$ was considered significant.

\section{Acknowledgments}

We thank Jon Roffey of Cancer Research Technology for AEPi synthesis; Nick Coe (University of Salford, Manchester, United Kingdom) for ASNase activity analyses on patient samples; Matthew Bogyo (Stanford University, Palo Alto, California, USA) for the AEP activity probe; Steve Bagley (Paterson Institute for Cancer Research) for help with microscopy; and Medac for the recombinant ASNase clone. The work was funded in part by a program grant from Cancer Research UK and a project grant from the Leukemia Research Fund (to V. Saha).

Received for publication November 5, 2008, and accepted in revised form April 8, 2009.

Address correspondence to: Vaskar Saha, Paterson Institute for Cancer Research, Wilmslow Road, Manchester M20 4BX, United Kingdom. Phone: 44-161-446-3094; Fax: 44-161-446-3092; E-mail: vaskar.saha@cancer.org.uk.

Catherine X. Moss's present address is: Wellcome Centre for Molecular Parasitology, University of Glasgow, Glasgow, United Kingdom.

Carly Leighton's present address is: Centre for Experimental Cancer Medicine, Queen Mary University of London, London, United Kingdom.

Frederik W. van Delft's present address is: Section of HaematoOncology, The Institute of Cancer Research, Brookes Lawley Building, Sutton, Surrey, United Kingdom.
1. Roy, A., et al. 2005. Early response to induction is predictive of survival in childhood Philadelphia chromosome positive acute lymphoblastic leukaemia: results of the Medical Research Council ALL 97 trial. Br. J. Haematol. 129:35-44.

2. Yang, L., et al. 2008. Asparaginase may influence dexamethasone pharmacokinetics in acute lymphoblastic leukemia. J. Clin. Oncol. 26:1932-1939.

3. Abshire, T.C., Pollock, B.H., Billett, A.L., Bradley, P., and Buchanan, G.R. 2000. Weekly polyethylene glycol conjugated L-asparaginase compared with biweekly dosing produces superior induction remission rates in childhood relapsed acute lymphoblastic leukemia: a Pediatric Oncology Group Study. Blood. 96:1709-1715.

4. Pession, A., et al. 2005. Long-term results of a randomized trial on extended use of high dose L-aspar- aginase for standard risk childhood acute lymphoblastic leukemia. J. Clin. Oncol. 23:7161-7167.

5. Moghrabi, A., et al. 2007. Results of the Dana-Farber Cancer Institute ALL Consortium Protocol 9501 for children with acute lymphoblastic leukemia. Blood. 109:896-904.

6. Asselin, B.L., et al. 1999. Prognostic significance of early response to a single dose of asparaginase in childhood acute lymphoblastic leukemia.J. Pediatr. Hematol. Oncol. 21:6-12.

7. Appel, I.M., et al. 2008. Pharmacokinetic, pharmacodynamic and intracellular effects of PEG-asparaginase in newly diagnosed childhood acute lymphoblastic leukemia: results from a single agent window study. Leukemia. 22:1665-1679.

8. Holleman, A., den Boer, M.L., Kazemier, K.M., Janka-Schaub, G.E., and Pieters, R. 2003. Resis- tance to different classes of drugs is associated with impaired apoptosis in childhood acute lymphoblastic leukemia. Blood. 102:4541-4546.

9. Muller, H.J., et al. 2000. Pegylated asparaginase (Oncaspar) in children with ALL: drug monitoring in reinduction according to the ALL/NHL-BFM 95 protocols. Br. J. Haematol. 110:379-384.

10. Zalewska-Szewczyk, B., et al. 2007. The anti-asparagines antibodies correlate with L-asparagines activity and may affect clinical outcome of childhood acute lymphoblastic leukemia. Leuk. Lymphoma. 48:931-936.

11. Avramis, V.I., and Panosyan, E.H. 2005. Pharmacokinetic/pharmacodynamic relationships of asparaginase formulations: the past, the present and recommendations for the future. Clin. Pharmacokinet. 44:367-393. 
12. Muller, H.J., et al. 2001. Pharmacokinetics of native Escherichia coli asparaginase (Asparaginase medac) and hypersensitivity reactions in ALL-BFM 95 reinduction treatment. Br. J. Haematol. 114:794-799.

13. Hak, L.J., et al. 2004. Asparaginase pharmacodynamics differ by formulation among children with newly diagnosed acute lymphoblastic leukemia. Leukemia. 18:1072-1077.

14. Stams, W.A., et al. 2003. Sensitivity to L-asparaginase is not associated with expression levels of asparagine synthetase in $\mathrm{t}(12 ; 21)+$ pediatric ALL. Blood. 101:2743-2747.

15. Appel, I.M., et al. 2006. Up-regulation of asparagine synthetase expression is not linked to the clinical response L-asparaginase in pediatric acute lymphoblastic leukemia. Blood. 107:4244-4249.

16. Fine, B.M., Kaspers, G.J., Ho, M., Loonen, A.H., and Boxer, L.M. 2005. A genome-wide view of the in vitro response to l-asparaginase in acute lymphoblastic leukemia. Cancer Res. 65:291-299.

17. Iwamoto, S., Mihara, K., Downing, J.R., Pui, C.H., and Campana, D. 2007. Mesenchymal cells regulate the response of acute lymphoblastic leukemia cells to asparaginase. J. Clin. Invest. 117:1049-1057.

18. Pinheiro, J.P., and Boos, J. 2004. The best way to use asparaginase in childhood acute lymphatic leukaemia--still to be defined? Br. J. Haematol. 125:117-127.

19. Asselin, B.L. 1999. The three asparaginases. Comparative pharmacology and optimal use in childhood leukemia. Adv. Exp. Med. Biol. 457:621-629.

20. Strefford, J.C., et al. 2006. Complex genomic alterations and gene expression in acute lymphoblastic leukemia with intrachromosomal amplification of chromosome 21. Proc. Natl. Acad. Sci. U. S. A. 103:8167-8172.

21. Watts, C., Matthews, S.P., Mazzeo, D., Manoury, B., and Moss, C.X. 2005. Asparaginyl endopeptidase: case history of a class II MHC compartment protease. Immunol. Rev. 207:218-228.

22. Antoniou, A.N., Blackwood, S.L., Mazzeo, D., and Watts, C. 2000. Control of antigen presentation by a single protease cleavage site. Immunity.
12:391-398.

23. Loak, K., et al. 2003. Novel cell-permeable acyloxymethylketone inhibitors of asparaginyl endopeptidase. Biol. Chem. 384:1239-1246.

24. Buttle, D.J., Murata, M., Knight, C.G., and Barrett, A.J. 1992. CA074 methyl ester: a proinhibitor for intracellular cathepsin B. Arch. Biochem. Biophys. 299:377-380

25. van Delft, F.W., et al. 2005. Prospective gene expression analysis accurately subtypes acute leukaemia in children and establishes a commonality between hyperdiploidy and $\mathrm{t}(12 ; 21)$ in acute lymphoblastic leukaemia. Br. J. Haematol. 130:26-35.

26. Chen, J.M., et al. 1997. Cloning, isolation, and characterization of mammalian legumain, an asparaginyl endopeptidase. J. Biol. Chem. 272:8090-8098.

27. van Zelm, M.C., et al. 2005. Ig gene rearrangement steps are initiated in early human precursor B cell subsets and correlate with specific transcription factor expression. J. Immunol. 175:5912-5922.

28. Werner, A., Rohm, K.H., and Muller, H.J. 2005. Mapping of B-cell epitopes in E. coli asparaginase II, an enzyme used in leukemia treatment. Biol. Chem. 386:535-540

29. Manoury, B., et al. 1998. An asparaginyl endopeptidase processes a microbial antigen for class II MHC presentation. Nature. 396:695-699.

30. Swain, A.L., Jaskolski, M., Housset, D., Rao, J.K., and Wlodawer, A. 1993. Crystal structure of Escherichia coli L-asparaginase, an enzyme used in cancer therapy. Proc. Natl. Acad. Sci. U. S. A. 90:1474-1478.

31. Aung, H.P., Bocola, M., Schleper, S., and Rohm, K.H. 2000. Dynamics of a mobile loop at the active site of Escherichia coli asparaginase. Biochim. Biophys. Acta. 1481:349-359.

32. Offman, M.N., Tournier, A.L., and Bates, P.A. 2008 Alternating evolutionary pressure in a genetic algorithm facilitates protein model selection. BMC Struct. Biol. 8:34.

33. Porter, C.T., Bartlett, G.J., and Thornton, J.M. 2004. The Catalytic Site Atlas: a resource of catalytic sites and residues identified in enzymes using structural data. Nucleic Acids Res. 32:D129-133.
34. Sexton, K.B., Witte, M.D., Blum, G., and Bogyo, M. 2007. Design of cell-permeable, fluorescent activity-based probes for the lysosomal cysteine protease asparaginyl endopeptidase (AEP)/legumain. Bioorg. Med. Chem. Lett. 17:649-653.

35. Li, D.N., Matthews, S.P., Antoniou, A.N., Mazzeo, D., and Watts, C. 2003. Multistep autoactivation of asparaginyl endopeptidase in vitro and in vivo. J. Biol. Chem. 278:38980-38990.

36. Jevnikar, Z., and Kos, J. 2008. CTSB (cathepsin B). Atlas Genet. Cytogenet. Oncol. Haematol. http://Atlas GeneticsOncology.org/Genes/CTSBID40202ch8p23. html.

37. Guo, M., Mathieu, P.A., Linebaugh, B., Sloane, B.F., and Reiners, J.J., Jr. 2002. Phorbol ester activation of a proteolytic cascade capable of activating latent transforming growth factor-betaL a process initiated by the exocytosis of cathepsin B. J. Biol. Chem. 277:14829-14837.

38. Wu, W., et al. 2006. Targeting cell-impermeable prodrug activation to tumor microenvironment eradicates multiple drug-resistant neoplasms. Cancer Res. 66:970-980.

39. Pui, C.H., Robison, L.L., and Look, A.T. 2008. Acute lymphoblastic leukaemia. Lancet. 371:1030-1043.

40. Mitchell, C.D., et al. 2005. Benefit of dexamethasone compared with prednisolone for childhood acute lymphoblastic leukaemia: results of the UK Medical Research Council ALL97 randomized trial. Br. J. Haematol. 129:734-745.

41. Vora, A., et al. 2006. Toxicity and efficacy of 6-thioguanine versus 6-mercaptopurine in childhood lymphoblastic leukaemia: a randomised trial. Lancet. 368:1339-1348.

42. Berman, H.M., et al. 2000. The Protein Data Bank. Nucleic Acids Res. 28:235-242.

43. Case, D.A., et al. 2006. AMBER 9. San Francisco: University of California. http://ambermd.org/ doc9/amber9.pdf.

44. Rizzari, C., et al. 2006. A pharmacological study on pegylated asparaginase used in front-line treatment of children with acute lymphoblastic leukemia. Haematologica. 91:24-31. 